\title{
Electromagnetic Scattering and Statistic Analysis of Clutter from Oil Contaminated Sea Surface
}

\author{
Cong-hui QI, Zhi-qin ZHAO \\ School of Electronic Engineering, University of Electronic Science and Technology of China, \\ Xiyuan Ave 2006, Chengdu, Sichuan, China \\ qiconghui0826@163.com, zqzhao@uestc.edu.cn
}

\begin{abstract}
In order to investigate the electromagnetic (EM) scattering characteristics of the three dimensional sea surface contaminated by oil, a rigorous numerical method multilevel fast multipole algorithm (MLFMA) is developed to preciously calculate the electromagnetic backscatter from the two-layered oil contaminated sea surface. Illumination window and resistive window are combined together to depress the edge current induced by artificial truncation of the sea surface. By using this combination, the numerical method can get a high efficiency at a less computation cost. The differences between backscatters from clean sea and oil contaminated sea are investigated with respect to various incident angles and sea states. Also, the distribution of the sea clutter is examined for the oilspilled cases in this paper.
\end{abstract}

\section{Keywords}

Oil contaminated sea, sea clutter, MLFMA, resistive loading

\section{Introduction}

Detection of oil spills is a long-term objective in sea remote sensing [1], [2]. The oil contaminated sea water is a threat to the marine environment. The vertical polarization of high frequency surface wave radar (HFSWR) has the feature of small energy attenuation, all weather operating and over-horizon. Most importantly, targets can be detected beyond the range of visibility. Thus HFSWR can be used to detect oil spills in far distance. When the sea water is contaminated by oil, the sea dynamics as well as the surface tension are changed [3]. Accordingly, the EM scattering characteristics from the oil contaminated sea surface are changed, such as radar echo and sea clutter distributions. The difference between backscatters from clean sea and oil contaminated sea is a useful feature and desirable in Synthetic Aperture Radar (SAR) imagery simulation of ocean scene which is often used to detect oil pollution on the ocean surface. Therefore, how to quantify the scattering differences is an open problem for the radar engineering.
Many researches have been made to examine the EM backscatter from the oil contaminated sea surface, but most of them are limited to two dimensional (2D) sea surface or focus on approximate method [3], [4]. However, the hypothetical 2D model lames their applications to three dimensional (3D) cases in practical engineering. Meanwhile, the oil contaminated sea surface is a two-layered dielectric problem. Therefore, the simulation in computational electromagnetics (CEM) becomes much complicate than the homogenous dielectric medium case, and needs further researches. It is our motivation.

In this paper, the electric field integral equation (EFIE) on the interfaces is derived using the wave equation combining the boundary condition. In order to exactly simulate the EM backscatter from the sea surface, the rigorous numerical multilevel fast multipole algorithm (MLFMA) [5], [6] is applied to obtain reliable results. In order to accelerate the EM simulation process when using MLFMA at low-grazing-angle (LGA) cases, a combination model of Thorsos illumination window [7] and resistive loading window [8] is applied to avoid the edge effect induced by artificial truncation of the sea surface in range and azimuth directions. These treatments can greatly improve the computation efficiency thus the simulation of the sea clutter is possible using relatively less computation resources. It is noted that this proposed method is more universal than these existing analytical methods, and unlimited by the $3 \mathrm{D}$ sea states or larger incident angles.

This rigorous numerical method and its treatments make the Monte Carlo simulation at LGA incidence possible. Then, the electromagnetic backscatter from the oil contaminated sea surfaces is computed with the variation of incident angles and sea states. As a comparison, the backscatter reduction and the statistic characteristics of the two kinds of sea clutter are analyzed compared with the clean sea surface. Some interesting conclusions are summarized in our experiments.

\section{Sea Model}

The sea surface can be generated using a spectral method which considers it as a superposition of harmonics. 
The amplitudes of the harmonics are proportion to a certain wind-dependent surface-roughness spectrum $W(K, \varphi)$. Then the sea surface can be obtained by inverse fast Fourier transform (IFFT). In this paper, the 2D PiersonMoskowitz (PM) spectrum is adopted to generate the 3D sea surface [9]. The PM spectrum is defined by

$$
W\left(K_{w}, \varphi\right)=\frac{\alpha}{2 K_{w}{ }^{4}} \exp \left\{-\frac{\beta g^{2}}{K_{w}{ }^{2} U^{4}}\right\} \cos ^{4}\left(\frac{\varphi-\varphi_{w}}{2}\right)
$$

where $K_{w}$ is the spatial wavenumber, $U$ is the wind speed at the height of $19.5 \mathrm{~m}$ above the sea surface, two constants $\alpha=8.1 \times 10^{-3}$ and $\beta=0.74$. The angle $\varphi$ is measured in the horizontal $x-y$ plane with respect to the $x$-axis and $\varphi_{w}$ is the wind direction. In our simulation, the wind direction is towards positive $x$-axis.

When the sea surface is contaminated by oil, the sea surface tension as well as the sea movement is changed. In the research of Lombardini et al. [10], it is demonstrated that the height of oil contaminated sea surface is damped comparing with the clean sea surface. This damping effect can be expressed by an attenuation coefficient, called Marngoni viscous damping coefficient [11]. In this paper, the oil layer thickness of the contaminated sea is $1 \mathrm{~mm}$.

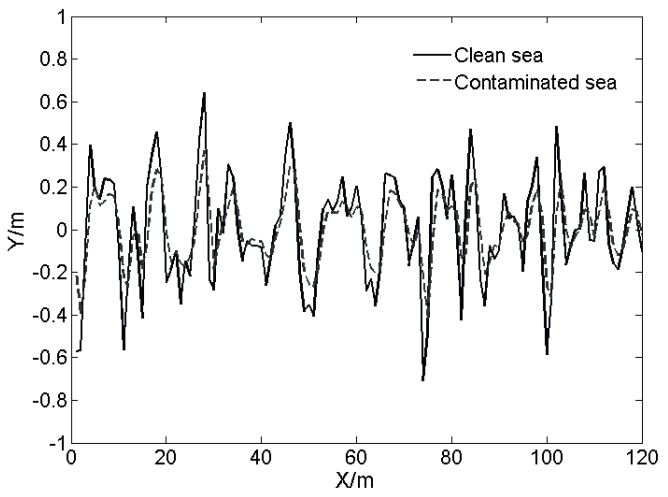

Fig. 1. Comparison of clean sea surface and contaminated sea surface.

Figure 1 shows the comparison of the geometrical profile between the clean sea surface and the contaminated sea surface. The simulation is performed at the wind speed of $U=7 \mathrm{~m} / \mathrm{s}$ which is considered as the average wind speed over the ocean. It is observed that the surface heights of the contaminated sea are indeed damped comparatively to the clean sea surface. Moreover, the small capillarityscale variations are strongly damped, which implies a strong damping of the surface slopes.

\section{EM Modeling}

Clean sea water is homogeneous dielectric medium with a relative permittivity $\varepsilon_{1}$ and permeability $\mu_{1}$. However, when the sea water is contaminated by oil whose relative permittivity is $\varepsilon_{2}$ and permeability is $\mu_{2}$ (generally,

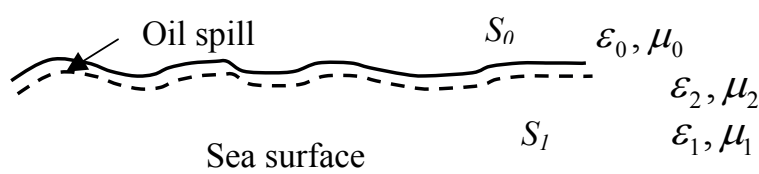

Fig. 2. Geometry of two-layered medium.

$\mu_{1}=\mu_{2}=1$ ), the contaminated sea surface becomes a twolayered dielectric material with its thickness $d$, as shown in Fig. 2.

Generally, the HFSW can work at LGA for some extreme cases. Therefore, the multipath effect is complicated for EM simulation. In this section, the rigorous numerical method MLFMA and its treatments are proposed to get the backscatters from the two-layered medium problem. The computation efficiency can be greatly improved, thus makes the Monte Carlo simulation of the sea surface possible.

\subsection{MLFMA}

Using the wave equation and the boundary conditions, EFIE can be derived as functions of the electric equivalent electric current $\boldsymbol{J}$ and equivalent magnetic current $\boldsymbol{M}$ which exist on the interface $S_{0}$ between the oil and air as well as the interface $S_{1}$ between oil and water. The two sets of surface currents on the boundaries $S_{0}$ and $S_{1}\left(\boldsymbol{J}_{0}, \boldsymbol{M}_{0}, \boldsymbol{J}_{1}\right.$, $\boldsymbol{M}_{1}$ ) can be determined using the following numerical simulation procedure.

$$
\begin{aligned}
& -i k_{0} Z_{0} \int_{S_{0}}\left\{\boldsymbol{J}_{0} G_{0}+\frac{1}{k_{0}^{2}} \nabla^{\prime} \cdot \boldsymbol{J}_{0} \nabla G_{0}\right\} d s^{\prime}+\oint_{S_{0}} \boldsymbol{M}_{0} \times \nabla G_{0} d s^{\prime}-\frac{1}{2} \boldsymbol{M}_{0} \\
& =-E^{i}, \vec{r} \in S_{0} \\
& i k_{1} Z_{1} \int_{S_{0}}\left\{\boldsymbol{J}_{0} G_{1}+\frac{1}{k_{1}^{2}} \nabla^{\prime} \cdot \boldsymbol{J}_{0} \nabla G_{1}\right\} d s^{\prime}-\oint_{S_{0}} \boldsymbol{M}_{0} \times \nabla G_{1} d s^{\prime} \\
& -i k_{1} Z_{1} \int_{S_{1}}\left\{\boldsymbol{J}_{1} G_{1}+\frac{1}{k_{1}^{2}} \nabla^{\prime} \cdot \boldsymbol{J}_{1} \nabla G_{1}\right\} d s^{\prime}+\oint_{S_{1}} \boldsymbol{M}_{1} \times \nabla G_{1} d s^{\prime}-\frac{1}{2} \boldsymbol{M}_{0}=0 \\
& \vec{r} \in S_{0}
\end{aligned}
$$

$i k_{1} Z_{1} \int_{S_{0}}\left\{\boldsymbol{J}_{0} G_{1}+\frac{1}{k_{1}^{2}} \nabla^{\prime} \cdot \boldsymbol{J}_{0} \nabla G_{1}\right\} d s^{\prime}-\oint_{S_{0}} \boldsymbol{M}_{0} \times \nabla G_{1} d s^{\prime}$

$-i k_{1} Z_{1} \int_{S_{1}}\left\{\boldsymbol{J}_{1} G_{1}+\frac{1}{k_{1}^{2}} \nabla^{\prime} \cdot \boldsymbol{J}_{1} \nabla G_{1}\right\} d s^{\prime}+\oint_{S_{1}} \boldsymbol{M}_{1} \times \nabla G_{1} d s^{\prime}-\frac{1}{2} \boldsymbol{M}_{1}=0$,

$\vec{r} \in S_{1}$

$-i k_{2} Z_{2} \int_{S_{0}}\left\{\boldsymbol{J}_{1} G_{2}+\frac{1}{k_{2}^{2}} \nabla^{\prime} \cdot \boldsymbol{J}_{1} \nabla G_{2}\right\} d s^{\prime}+\oint_{S 1} \boldsymbol{M}_{1} \times \nabla G_{2} d s^{\prime}-\frac{1}{2} \boldsymbol{M}_{1}$ $=0, \vec{r} \in S_{1}$

where $\oint_{S_{0}} d s^{\prime}$ denotes principle integration, $Z_{n}$ is the characteristic impedance, $k_{n}$ is the wave number and 
$G_{n}=g_{n}\left(r, r^{\prime}\right)=4 \pi e^{i k_{n}\left|r-r^{\prime}\right|} /\left|r-r^{\prime}\right|$ is the Green function. The subscript $n=1,2,3$ denotes the three cases in free space, sea water and oil medium, respectively.

Here the equivalent impedance boundary condition is used to deal with the boundary condition scattering problem [12]. It means the electric current $\boldsymbol{J}$ and equivalent magnetic current $\boldsymbol{M}$ satisfy $n^{\prime} \times \boldsymbol{M}_{0}=Z_{1} \boldsymbol{J}_{0}$ and $n^{\prime} \times \boldsymbol{M}_{1}=Z_{2} \boldsymbol{J}_{1}$, where $n^{\prime}$ is the outer normal vector at each field point. Therefore, the unknown number in (2) will be halved. The unknown equivalent electric current can be obtained by the Galerkin's method in method of moment (MoM), as following.

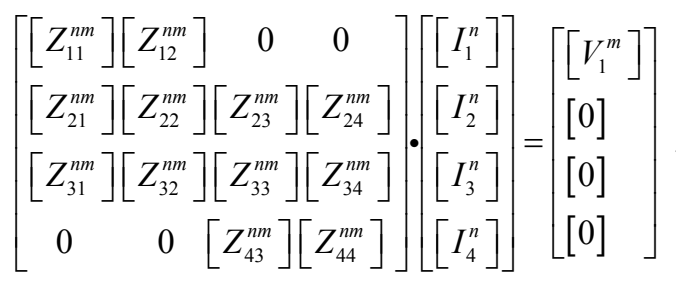

To investigate the statistic characteristics of the backscatter of the sea surface, Monte Carlo simulation is necessary. However, it is rather computationally expensive and time-consuming. In order to accelerate the computing process, MLFMA is used to solve the above equation. In the process of MLFMA, the interactions between the elements are classified as near-region and the far-region. The nearregion matrix elements are calculated directly using MoM, while the far-region elements are acquired by using MLFMA.

$$
\sum_{l} Z^{n m} I^{n}=\sum_{l \in N R} Z^{n m} I^{n}+\sum_{l \in F R} Z^{n m} I^{n}
$$

where $N R$ represents the near-region and $F R$ represents the far-region, $l$ means the basis function, $I^{n}$ are the expansion coefficients needed to solve using MoM, and $Z^{n m}$ are the elements of the impedance matrix. More details can be referenced in [5].

\subsection{Treatments}

Numerical methods can guarantee sufficient precision when solving EM computing problems even at LGA. However, it will bring edge diffraction which is induced by the artificial truncation to the sea surface. The electric current $\boldsymbol{J}$ will change suddenly at the edge of the truncated surface. This phenomenon is called edge effect. In the research of EM scattering in those numerical methods, how to avoid the edge effect is an important issue. The most popular way to suppress the edge effect is using the illumination tapered window (Thorsos window) [7] which is got by modulating a tapered function to incident electric field, as following.

$$
p(x, z)=e^{-j \frac{2\left(x-z \tan \theta_{i}\right)^{2} / g^{2}-1}{\left(k g \cos \theta_{i}\right)^{2}} k \hat{k} \cdot r^{\prime}} e^{-\frac{\left(x-z \tan \theta_{i}\right)^{2}}{g^{2}}}
$$

where $g$ is a constant that controls the width of the illumination beam, $\theta_{i}$ is the incident angle.

However, according to the studies by Jin [13], the length $L$ of rough surface has to satisfy the condition: $L \geq 24 \lambda /\left(\cos \theta_{i}\right)^{1.5}$, where $\lambda$ is the EM wavelength. Accordingly, at LGA the scale of the computing sea surface is very large. For example, if $\theta_{i}=85^{\circ}$, then $L \geq 932 \lambda$. And it leads to a large amount of unknown number on the sea surface. Therefore, instead of the illumination window, the resistive loading window is applied to suppress the edge effect under the plane wave illumination. It can be accomplished by simply adding $R \boldsymbol{J}$ to the EFIE formula, taking (2a) as an example, as following

$$
\overline{\bar{L}}(\boldsymbol{J})+R \boldsymbol{J}=-E^{i}, \vec{r} \in S_{0}
$$

where $R$ is the resistance, and $\overline{\bar{L}}(\cdot)$ is the matrix-vector multiply operator and denotes the procedure in left side of (2a). The resistance is loaded within the resistive loading area, which was discussed in detail in reference [14]. Once the resistive loading method is used, the length $L$ of sea surface only needs to be as large as 10 times of the correlation length of the rough surface. It is much smaller than the condition of Thorsos window case.

However, it is found that the resistive loading will bring the convergence problem because that the impedance matrix $Z^{n m}$ is a non-diagonally dominant matrix for this two-layered dielectric medium scattering problem in our simulations. Since the resistance $R$ will attenuate the value of self-impedance element, the condition number of the final discretized impedance matrix will become worse. Therefore, more computational CPU time will be taken in the impedance matrix iteration processes.

A method combining the two different windows is proposed to accelerate its convergence. Resistive loading window is applied in range direction while a Gaussian illumination window is used in azimuth direction. In azimuth direction, the incident plane wave is modulated by a Gaussian function $p(y)=e^{-y^{2} / g^{2}}$ instead of the Thorsos window. Meanwhile, an important work in range direction is to extend the original sea surface with smoothly curved sections (for example, we used radius of $10 \lambda$ ) that join to planar sections which are angled $30^{\circ}$ down from the horizon. So that all the points on the extension surfaces are shadowed by the original rough surface. The resistive loading of the edges has the advantage of not requiring the surface to be modified at LGA.

As a comparison, a $600 \mathrm{~m}(L) \times 600 \mathrm{~m}(W)$ PM sea surface realization at $U=3 \mathrm{~m} / \mathrm{s}$ was generated and simulated under those system parameters: radar frequency $15 \mathrm{MHz}$, incident angle $60^{\circ}$, vertical polarization. As shown in Fig. 4, the convergence iteration is 228 steps for the traditional resistive window [8] in range and azimuth directions whereas only 50 steps are needed for the proposed combination of the two different windows. 


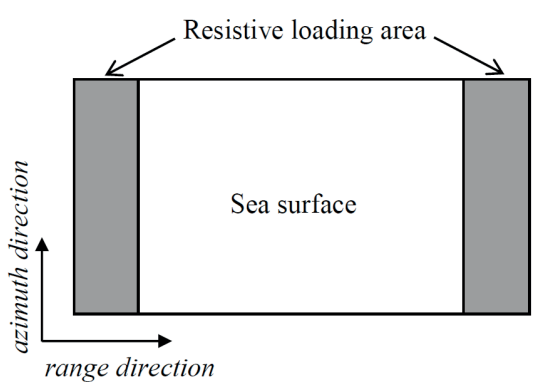

Fig. 3. The scheme of two windows.

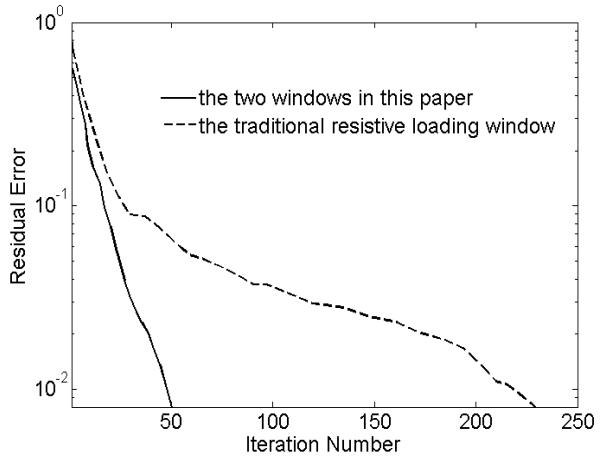

Fig. 4. Convergence comparison.

Therefore, the proposed method to suppress the edge effect is more effective and can greatly reduce the CPU time, which is a big improvement in Monte Carlo simulation.

\section{Numerical Simulations}

\subsection{Comparison of Backscatters}

By using this proposed method, some experiments are presented in this section. The HFSWR operates at the frequency of $15 \mathrm{MHz}$, vertical polarization. Monte Carlo simulation is applied to get average radar backscatter. A total number of 1000 sea surfaces are generated for each sea state. The simulation sea area is $600 \mathrm{~m}$ long with a width of $600 \mathrm{~m}$. The simulation is performed when wind speed is $3 \mathrm{~m} / \mathrm{s}$ and $9 \mathrm{~m} / \mathrm{s}$, corresponding to Douglas sea state 2 and 4 .

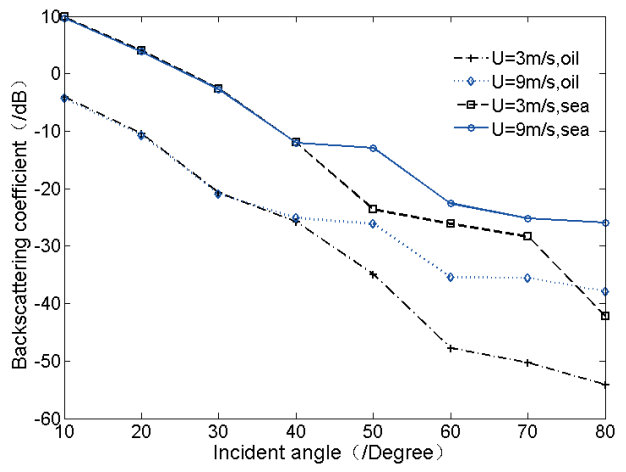

Fig. 5. Backscatter coefficients with various wind speeds.

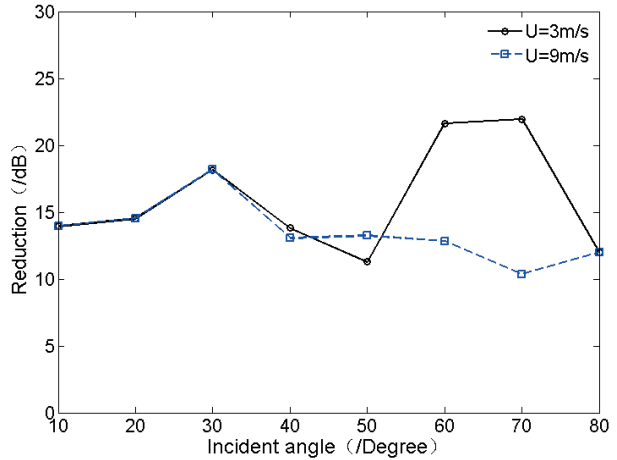

Fig. 6. Backscatter coefficient reduction.

As shown in Fig. 5, the radar backscatters of the contaminated sea surface is much lower than that of the clean sea surface. The reduction of the backscatter coefficient corresponding to different sea states is shown in Fig. 6. A reduction of more than $10 \mathrm{~dB}$ at moderate incident angles is found through our simulation, and even $15 \mathrm{~dB}$ at LGA angle. In SAR systems, the oil polluted area is seen as "dark" sea. Capillary waves on the sea surface reflect electromagnetic energy. However, when the oil layer on the sea surface, the capillary waves are dampened. As a consequence, the backscatter is much weaker than clean sea surface.

\subsection{Sea Clutter Distribution}

By using the radar echo data acquired in the above experiments, the distribution of the sea clutter is also examined. For radar system, statistical models (Rayleigh, Lognormal, Weibull and K-distributions) are often used to describe the sea clutter. In this paper, the probability density functions (PDF) of the backscatter of the sea clutter for clean sea and oil-contaminated sea are studied. The parameters of Rayleigh, Lognormal and Weibull distribution are estimated by the maximum likelihood (ML) method. It is a standard approach to parameter estimation and provides optimum estimates in the sense that these estimates are the most probable parameter values. However, ML is computationally expensive to estimate the parameters of $\mathrm{K}$ distribution. A new method based on higher order and fractional moments is proposed in these references [15], [16]. It can significantly reduce the computational requirement. Details about the formulas of these four distributions and the estimation methods are summarized in reference [16]. Take this sea state of $U=9 \mathrm{~m} / \mathrm{s}$ as an example, the sea clutter distribution is shown for different incident angles in Fig. 7.

Kolmogorov-Smirnov test (K-S test) is used to find the best fitting distribution of the sea clutter among the four distributions. K-S test has the advantage of making no assumption about the distribution of the sea data. It is nonparametric and distribution free. The K-S test statistic quantifies a distance between the empirical distribution function of the sample and the cumulative distribution function of the reference distribution, or between the 


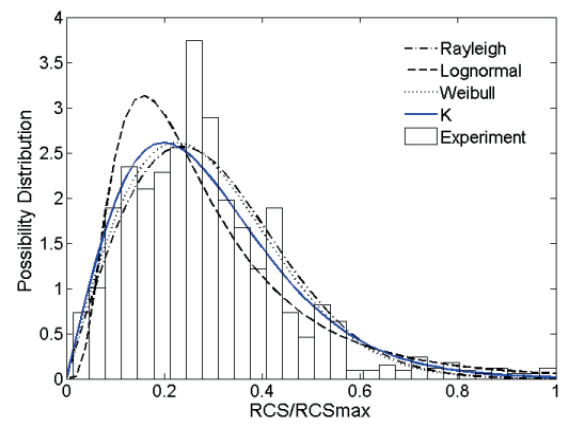

(a) Clean sea surface at incident angle of $30^{\circ}$

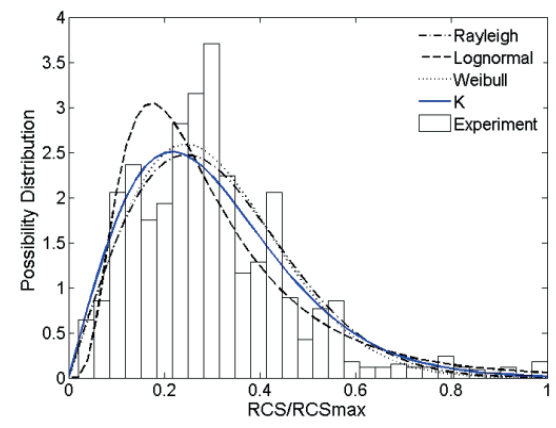

(b) Oil contaminated sea at incident angle of $30^{\circ}$

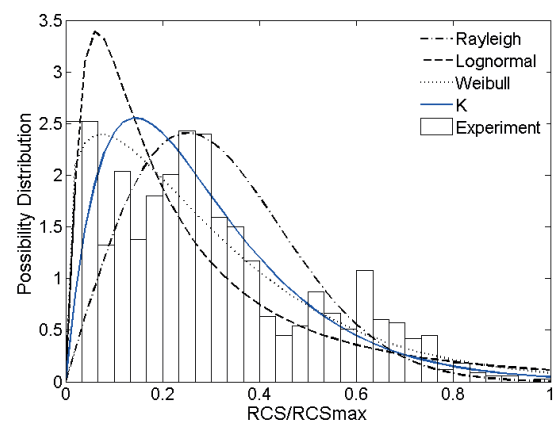

(c) Clean sea at incident angle of $80^{\circ}$

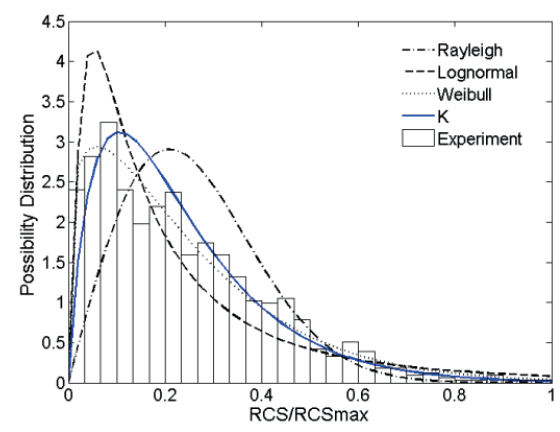

(d) Oil contaminated sea at incident angle of $80^{\circ}$

Fig. 7. The backscatter distribution of sea clutter.

empirical distribution functions of two samples. The K-S test statistic is defined as $D e$ which is shown

$$
D e=\max _{1 \leq i \leq N}\left|F\left(x_{i}\right)-F_{t}\left(x_{i}\right)\right|=\max _{1 \leq i \leq N}\left|n / N-F_{t}\left(x_{i}\right)\right| .
$$

$F_{t}\left(x_{i}\right)$ is the cumulative distribution of the distribution being tested and $F\left(x_{i}\right)$ is the cumulative distribution of the simulated sea clutter. De means the maximum deviation between the cumulative distribution of the tested distribution and the statistic distribution of the simulation result. The best fit distribution is with the smallest $D e$. Tab. 1 is the K-S test of the sea clutter distribution corresponding to Fig. 7. And the sea clutter distributions are also examined under different sea states in Tab. 2 and Tab. 3.

\begin{tabular}{|c|c|c|c|c|}
\hline$D e$ & Rayleigh & Lognormal & Weibull & $K$ \\
\hline Sea $/ 30^{\circ}$ & $\mathbf{0 . 0 4 1 9}$ & 0.1140 & 0.0451 & 0.0597 \\
\hline Sea $/ 80^{\circ}$ & 0.1281 & 0.1446 & $\mathbf{0 . 0 6 1 4}$ & 0.0914 \\
\hline Oil $/ 30^{\circ}$ & 0.0493 & 0.1156 & $\mathbf{0 . 0 4 4 4}$ & 0.0609 \\
\hline Oil $/ 80^{\circ}$ & 0.1530 & 0.1106 & $\mathbf{0 . 0 3 9 7}$ & 0.0566 \\
\hline
\end{tabular}

Tab. 1. K-S test.

\begin{tabular}{|c|c|c|c|c|c|c|c|c|}
\hline Incident angle & $10^{\circ}$ & $20^{\circ}$ & $30^{\circ}$ & $40^{\circ}$ & $50^{\circ}$ & $60^{\circ}$ & $70^{\circ}$ & $80^{\circ}$ \\
\hline State $2(3 \mathrm{~m} / \mathrm{s})$ & $\mathrm{L}$ & $\mathrm{K}$ & $\mathrm{K}$ & $\mathrm{L}$ & $\mathrm{W}$ & $\mathrm{W}$ & $\mathrm{L}$ & $\mathrm{W}$ \\
\hline State $4(9 \mathrm{~m} / \mathrm{s})$ & $\mathrm{K}$ & $\mathrm{R}$ & $\mathrm{W}$ & $\mathrm{W}$ & $\mathrm{W}$ & $\mathrm{W}$ & $\mathrm{W}$ & $\mathrm{W}$ \\
\hline
\end{tabular}

Tab. 2. The distribution of sea clutter for contaminated sea.

\begin{tabular}{|l|c|c|c|c|c|c|c|c|}
\hline Incident angle & $10^{\circ}$ & $20^{\circ}$ & $30^{\circ}$ & $40^{\circ}$ & $50^{\circ}$ & $60^{\circ}$ & $70^{\circ}$ & $80^{\circ}$ \\
\hline State $2(3 \mathrm{~m} / \mathrm{s})$ & $\mathrm{K}$ & $\mathrm{K}$ & $\mathrm{K}$ & $\mathrm{L}$ & $\mathrm{W}$ & $\mathrm{L}$ & $\mathrm{L}$ & $\mathrm{W}$ \\
\hline State $4(9 \mathrm{~m} / \mathrm{s})$ & $\mathrm{K}$ & $\mathrm{K}$ & $\mathrm{R}$ & $\mathrm{W}$ & $\mathrm{W}$ & $\mathrm{W}$ & $\mathrm{W}$ & $\mathrm{W}$ \\
\hline
\end{tabular}

Tab. 3. The distribution of sea clutter for clean sea.

As can be seen from Tab. 2 and Tab. 3, the distribution of clutter from oil contaminated sea surface is different from that of clean sea surface. For most cases, the sea clutter satisfies $\mathrm{K}$ distribution and Weibull distribution. When the wind speed above the sea surface is moderate $(3 \mathrm{~m} / \mathrm{s})$, $\mathrm{K}$ distribution describes the sea clutter quite well at small incident angle. As the incident angle getting large, it is Weibull distribution for the contaminated sea whereas it is Lognormal distribution for the clean sea. When the wind speed gets higher $(9 \mathrm{~m} / \mathrm{s})$, Weibull distribution will be dominant at LGA. The distribution of clutter from oil contaminated sea is first mentioned in this paper. Rigorous numerical EM simulation method can ensure sufficient accuracy. Moreover, with the treatments this method can obtain a high efficiency. Consequently, Monte Carlo simulation can be performed on the electric large problem. The real radar data from HFWSR system is usually very expensive. Instead of the real data from the radar, the simulation results by using rigorous numerical computational electromagnetic method as shown in this paper is workable as well. Moreover, it is more cost-effective and available.

\section{Conclusions}

In this paper, the sea clutter distribution is examined when there is oil pollution on the sea surface. In HFSWR system, the observation area is very large; accordingly the accurate EM simulation of the large sea surface is rather difficult. The integral equations both on the clean sea surface and the oil contaminated sea surface are presented. Numerical method with treatments on it can ensure suffi- 
cient accuracy to get reliable results of the backscatter from sea surface. Meanwhile, the computational resources are saved a lot, and then Monte Carlo simulation can be performed. It is found that the backscatter from oil contaminated sea surface is much weaker than that from clean sea surface. That is because the oil damped the capillary waves. Accordingly, the reflected electromagnetic energy is weaker than before. This effect can be seen in SAR image that the oil contaminated area is dark. The distribution of the sea clutter which is an important statistic characteristic is investigated by using the simulated results. Differences of the sea clutter distribution are found between the oil contaminated sea surface and the clean sea surface.

\section{Acknowledgements}

This work was supported by the National Natural Science Foundation of China under grant 61171044; the Fundamental Research Funds for the Central Universities under grant ZYGX2012YB006; and Research Fund for the Doctoral Program of Higher Education of China under grant 20120185110024.

\section{References}

[1] SOLBERG, A. H. S. Remote sensing of ocean oil-spill pollution. Proceedings of the IEEE, 2012, vol. 100, no. 10, p. 2931-2945. DOI: 10.1109/JPROC.2012.2196250

[2] FINGAS, M., BROWN, C. Review of oil spill remote sensing. Marine Pollution Bulletin, 2014. DOI: 10.1016/S13532561(98)00023-1

[3] PINEL, N., DECHAMPS, N., BOURLIER, C. Modelling of the bistatic electromagnetic scattering from sea surfaces covered in oil for microwave applications. IEEE Transactions on Geoscience and Remote Sensing, 2008, vol. 46, no. 2, p. 385-392. DOI: 10.1109/TGRS.2007.902412

[4] GHANMI, H., KHENCHAF, A., COMBLET, F. Numerical modeling of electromagnetic scattering from sea surface covered by oil. Journal of Electromagnetic Analysis and Applications, 2014, vol. 6, p. 15-24. DOI: 10.4236/jemaa.2014.61003

[5] SONG, J., LU, C.-C., CHEW, W. C. Multilevel fast multipole algorithm for electromagnetic scattering by large complex objects. IEEE Transactions on Antennas and Propagation, 1997, vol. 45, no. 10 , p. 1488-1493. DOI: $10.1109 / 8.633855$

[6] YANG, W., ZHAO, Z., QI, C., NIE, Z. Electromagnetic modeling of breaking waves at low grazing angles with adaptive higher order hierarchical Legendre basis functions. IEEE Transactions on Geoscience and Remote Sensing, 2011, vol. 49, no. 1, p. 346-352. DOI: $10.1109 /$ TGRS.2010.2052817

[7] THORSOS, E. I. The validity of the Kirchhoff approximation for rough surface scattering using a Gaussian roughness spectrum. Journal of the Acoustical Society of America, 1988, vol. 83, p. 78 to 92. DOI: DOI: 10.1121/1.396188
[8] ZHAO, Z., WEST, J. C. Resistive suppression of edge effects in MLFMA scattering from finite conductivity surfaces. IEEE Transactions on Antennas and Propagation, 2005, vol. 53, p. 1848-1852. DOI: 10.1109/TAP.2005.846810

[9] LI, X., XU, X. Scattering and Doppler spectral analysis for twodimensional linear and nonlinear sea surfaces. IEEE Transactions on Geoscience and Remote Sensing, 2011, vol. 49, p. 603-611. DOI: $10.1109 /$ TGRS.2010.2060204

[10] LOMBARDINI, P., Fiscella, B., TRIVERO, P., CAPPA, C., GARRETT, W. Modulation of the spectra of short gravity waves by sea surface films: slick detection and characterization with a microwave probe. Journal of Atmospheric and Oceanic Technology, 1989, vol. 6, p. 882-890. DOI: 10.1175/15200426(1989)006<0882:MOTSOS>2.0.CO;2

[11] GADE, M., ALPERS, W., HUHERFUSS, H., WISMANN, V. R., LANGE, P. A, On the reduction of the radar backscatter by oceanic surface films: Scatterometer measurements and their theoretical interpretation. Remote Sensing of Environment, 1998, vol. 66, p. 52-70. DOI:10.1016/S0034-4257(98)00034-0

[12] GLISSON A. W. Electromagnetic scattering by arbitrarily shaped surface with impedance boundary conditions. Radio Science, 1992, vol. 27, no. 6, p. 935-943. DOI: 10.1029/92RS01782

[13] LIU, P., JIN, Y. Q. Numerical simulation of bistatic scattering from a target at low altitude above rough sea surface under an EMwave incidence at low grazing angle by using the finite element method. IEEE Transactions on Antennas and Propagation, 2004, vol. 52, no. 5, p. 1205-1210. DOI: 10.1109/TAP.2004.827497

[14] ZHAO, Z., WEST, J. C. Resistive treatment of edges in MLFMA LGA scattering from finite conductivity 2D surfaces. In IEEE Antennas and Propagation Society International Symposium, 2002, p. 264-267. DOI: 10.1109/APS.2002.1016974

[15] ISKANDER, D. R., ZOUBIR, A. M. Estimation of the parameters of the K-distribution using higher order and fractional moments [radar clutter]. IEEE Transactions on Aerospace and Electronic Systems, 1999, vol. 35, p. 1453-1457. DOI: 10.1109/7.805463

[16] FARINA, A., GINI, F., GRECO, M., VERRAZZANI, L. High resolution sea clutter data: statistical analysis of recorded live data. IEE Proceedings-in Radar, Sonar and Navigation, 1997, vol. 144, no. 3, p. 121-130. DOI: 10.1049/ip-rsn:19971107

\section{About the Authors ...}

Cong-hui QI was born in Hebei, China. She received her M.Sc. from University of Electronic Science and Technology of China in 2009. Her research interests include computational electromagnetic, backscatter from rough surface and SAR image.

Zhi-qin ZHAO was born in Hunan, China. He received B.S. and M.S. degrees in Electronic Engineering from the University of Electronic Science and Technology of China, Sichuan, and the Ph.D. degree in Electrical Engineering from Oklahoma State University, Stillwater, in 1990, 1993, and 2002, respectively. His research interests include radar signal processing and computational electromagnetics. 